\title{
La escritura creativa en la era digital: el foro de rol como herramienta de aprendizaje en $\mathbf{E} / \mathbf{L E}$
}

\author{
MARÍA MOLÉS MORENO \\ Universitat de València \\ momoma2@alumni.uv.es
}

\begin{abstract}
Resumen: El presente artículo investiga el potencial de los foros de rol como recurso de aprendizaje y desarrollo de la destreza escrita en E/LE, en especial la expresión escrita, con el objetivo de reemplazar las actividades más tradicionales de composición creativa por herramientas más innovadoras y, por tanto, aproximar las TIC a la realidad de la enseñanza de E/LE. Con este fin, se ha realizado una investigación acerca del objeto de estudio, los foros de rol, y se han estudiado sus características - funcionalidad, mecánicas, comportamientos, motivación, etc.- por medio de un trabajo de campo. A continuación, a partir de las conclusiones de la investigación previa, se han expuesto las ventajas y los posibles inconvenientes de esta herramienta dentro del aula de E/LE.
\end{abstract}

Palabras clave: destreza escrita, escritura creativa, TIC, foros, rol, juegos de rol

\section{Creative Writing In Digital Era: RPG Forums As A Learning Tool In SFL}

Abstract: This paper investigates the potential of RPG (role-playing games) forums as a learning resource to improve writing and reading skills, this study however is specifically writing focused. This investigation aims to replace oldfashioned creative writing activities with more innovative tools and therefore it tries to unite new technologies with a more hands on learning experience. To achieve this, the core study object, RPG forums, has been examined through a field research focusing on its reality - function, mechanics, user behavior, motivations, etc). Finally, based on the conclusions of the previous research, the advantages and possible disadvantages of this tool within the SFL classroom have been explained.

Key words: written production, creative writing, new technologies, forums, role, roleplaying

\section{Introducción}

En un mundo en el que el progreso se encuentra íntimamente ligado al desarrollo de la tecnología y de la cultura digital, resulta indiscutible que los avances en cualquier ámbito de nuestra sociedad no pueden permanecer al margen de la realidad digital en la que vivimos. La educación, y por ende la enseñanza de E/LE, no son una excepción.

Tal ha sido esta revolución que, por ejemplo, el nuevo MCER ya recoge dentro de las diferentes actividades de interacción, además de las orales y escritas, una nueva modalidad: las interacciones en línea (2018: 83-98). El presente artículo trata de abogar por la incorporación de nuevas prácticas docentes y del uso de nuevas herramientas 
digitales dentro de la enseñanza de lenguas extranjeras; por ello, presentamos los foros de rol como plataforma innovadora con la que trabajar dentro del aula.

Desde una perspectiva idílica de la enseñanza de E/LE, el docente conduciría las sesiones de manera que cada una de las destrezas lingüísticas pudiera ser atendida de manera equivalente. Sin embargo, la realidad es otra: tradicionalmente, de entre todas las destrezas, la producción escrita tiende a ser aquella más olvidada, ocupando un lugar secundario dentro del aula de idiomas. Existen diferentes factores que pueden haber influido en la degradación de la producción escrita, como la complejidad de abarcar aspectos como los procesos de composición en una misma tarea (Cassany, 1989: 64-66) o el actual predominio del enfoque comunicativo dentro de la enseñanza de idiomas y el consecuente auge de las destrezas orales como práctica prioritaria por considerarlas más próximas a y, de alguna manera, más necesarias para la vida cotidiana.

No obstante, si tenemos presente la nueva era digital en la que vivimos y que ya señalábamos al inicio de este epígrafe, podemos observar una verdadera revolución de los textos escritos: a lo largo de nuestro día a día, consumimos y producimos una elevada cantidad de escritos a través de nuevos canales y plataformas de comunicación digital, por lo que se vuelve esencial incorporar la destreza escrita en la enseñanza de E/LE y, al mismo tiempo, dar con nuevas aproximaciones y propuestas didácticas que logren introducir esta realidad digital en el aula.

Tradicionalmente, la producción escrita ha abierto diferentes interrogantes para los docentes a la hora de incluirla dentro de un plan curricular. Una de las cuestiones principales se centra en la elección del tema, pues supone una decisión que preocupa en particular a los docentes (Raimes, 1991: 413), probablemente debido a que existe un especial cuidado en orientar toda acción dentro del aula hacia un fin comunicativo específico, una tendencia influenciada por el enfoque comunicativo. Con este artículo pretendemos también romper una lanza a favor de aquellas prácticas escritas que tal vez no persiguen un fin tan comunicativo como preparar al aprendiente para una entrevista de trabajo, pero que pueden favorecer su creatividad y su mundo interior, así como incentivar su motivación hacia el aprendizaje.

En este último aspecto, a menudo conciliar el aprendizaje con la motivación no resulta tarea fácil, sobre todo cuando tratamos con una destreza tan compleja como puede ser la escrita. Por esta razón, consideramos fundamental analizar y comprender qué clase de producciones escritas y creativas se realizan dentro de esta realidad digital de la que hablamos con el fin de adaptarlas y trasladarlas al campo de la enseñanza de E/LE.

\section{La escritura creativa en la cultura digital}

\subsection{Los orígenes de los foros de rol: la fanfiction y los juegos de rol}

A lo largo de las últimas décadas, la revolución digital ha dado paso a nuevas formas de interacción, como ya veníamos anunciando, así como también ha permitido la creación de plataformas virtuales mediante las que establecer esta comunicación. Uno de los recursos que más útiles han demostrado ser dentro del campo de la educación, en especial para cursos a distancia, son los foros de discusión. Estas páginas se definen como espacios en línea a través de los que varios participantes intercambian opiniones y emulan, de manera virtual, los debates que se realizan en foros presenciales.

Los foros de discusión presentan una serie de características que han demostrado ser especialmente útiles dentro del campo de la educación a distancia, puesto que promueven 
una interacción escrita completamente asincrónica, permitiendo que los participantes se encuentren en lugares y momentos completamente distintos. No obstante, los foros disponen de otras ventajas que los convierten en una herramienta con un gran potencial para la enseñanza. En concreto, centraremos el foco de este estudio en los foros de rol, una vertiente alternativa en el uso de este tipo de plataformas con una mayor carga creativa.

De manera previa a la profundización del objeto de estudio, resulta adecuado realizar una contextualización del espacio en el que surgen este tipo de páginas, de forma que también nos permita entender las características de esta modalidad de rol tan peculiar. Toda esta serie de plataformas virtuales que señalábamos anteriormente propició también el nacimiento de nuevos géneros textuales a través de Internet, un espacio de dimensiones y conexiones completamente transformadoras en el que cualquier individuo puede producir su propio texto y lograr llegar a una cantidad notable de consumidores. Dentro de este espacio nace la fanfiction, un caso muy reciente de producción escrita que surge como respuesta a una obra - literaria, cinematográfica o de otras características - ya existente. Como su propio nombre indica, la fanfiction se trata de una producción escrita realizada por fans y para fans que se aprovecha, en un buen sentido, de todo un universo creado anteriormente, con unos personajes, lugares y demás elementos ya conocidos; con toda esta información sobre la mesa, los escritores de fanfiction han creado nuevos textos a partir de la obra original con la única finalidad de disfrutar del universo del que son aficionados $\mathrm{y}$, al mismo tiempo, entretener a otros.

De forma paralela, y con el fin de comprender el origen de los foros de los que hablaremos a continuación, resulta pertinente conocer qué se entiende exactamente por un juego de rol. Este tipo de actividad de entretenimiento consiste, por un lado, en la interpretación de un personaje; cada participante adopta un rol determinado y actúa en función de las particularidades que caracterizan a su personaje. Para desarrollar el juego, los personajes interactúan a lo largo de una aventura ficticia, siguiendo unas reglas establecidas con anterioridad y habitualmente bajo la supervisión y guía de un narrador o master.

Así pues, para abordar nuestro objeto de estudio, hemos de tener muy presentes las dos vertientes que hicieron posible su aparición: por una parte, la actividad que se produce en un foro de rol está íntimamente relacionada a la lectura y, sobre todo, la escritura de sus usuarios sobre una ambientación determinada, tal y como ocurre en la fanfiction; pero, a su vez, también se manifiestan en su desarrollo diferentes características más típicas de un juego, puesto que bebe de las particularidades de los juegos de rol de tablero. En nuestra investigación, debido a nuestro interés lingüístico y didáctico, el foco de atención se encuentra más localizado sobre el primer aspecto, que nos permite realizar una explotación de la destreza escrita.

\subsection{Una nueva forma de narración: la mecánica play-by-post-roleplaying}

Una vez conocidos estos primeros inicios sobre la escritura creativa en el mundo digital, podemos afirmar que es precisamente en los foros de rol donde parecen converger este nuevo género textual y las actividades recreativas de rol. Al contrario de los foros más genéricos, un foro de rol no es una página de debate, sino de creación; en él, los usuarios establecen una serie de conexiones que les llevan a desarrollar de manera conjunta una misma historia.

Tal y como ocurre con la fanfiction, cada foro de rol se crea atendiendo a las necesidades de la ambientación escogida, según la obra original a la que, de alguna forma, homenajea. 
El diseño, la estructura y la distribución de las categorías se verán especialmente marcados por esta temática. Las categorías - las distintas secciones que conforman el foro- se convertirán en los escenarios de rol, dependiendo de la ambientación ficticia determinada: mundos, países, lugares, etc.; en un foro basado en el mundo de Canción de hielo y fuego, las categorías serán completamente diferentes a las de un foro inspirado en las historias de los cómics de Marvel. Por ejemplo, en el caso que planteamos de la saga de Canción de hielo y fuego, las diferentes categorías aludirán a las localizaciones que aparecen en la historia: el Norte, Dorne, el Dominio, etc.

Dentro de la plataforma, cada usuario se encarga de interpretar a un personaje - es decir, lo rolea - dentro del universo creado en la plataforma. Para establecer un punto de partida y facilitar que el resto de participantes conozcan a cada personaje, el usuario debe completar y publicar una ficha de su personaje que recoja la información y los datos más importantes de su personaje. Normalmente el modelo a completar de esta ficha se proporciona por parte de la administración, de modo que todas las fichas de personajes sigan un esquema común dentro del mismo foro.

A pesar de todas las diferencias dependiendo de la ambientación o del tipo de personajes, el funcionamiento de los foros de rol suele responder a una misma mecánica; este tipo de páginas, que se benefician de las características propias de los foros virtuales, han desarrollado una mecánica de comunicación y de creación que las caracterizan por completo.

Las producciones escritas del foro se construyen de modo colaborativo entre dos o más participantes, cada uno interpretando a un personaje diferente, y se van publicando a través de mensajes, dando lugar a la mecánica play-by-post-roleplaying; es decir, la historia ficticia que se establece avanza necesariamente con la colaboración de otros personajes, permitiendo que estos interactúen y se relacionen a través de temas o hilos ${ }^{1}$. Esta mecánica permite que los usuarios escriban una especie de reacción al mensaje que el otro participante publicó con anterioridad y permiten que la historia esté realmente viva.

\footnotetext{
${ }^{1}$ Cadenas de mensajes que, normalmente, abarcan una escena en cuestión entre dos o más personajes; son el equivalente más próximo a lo que consideraríamos un capítulo dentro de una novela. Los hilos quedan registrados dentro de las diferentes categorías del foro, dependiendo del escenario ficticio en el que se desarrollan.
} 


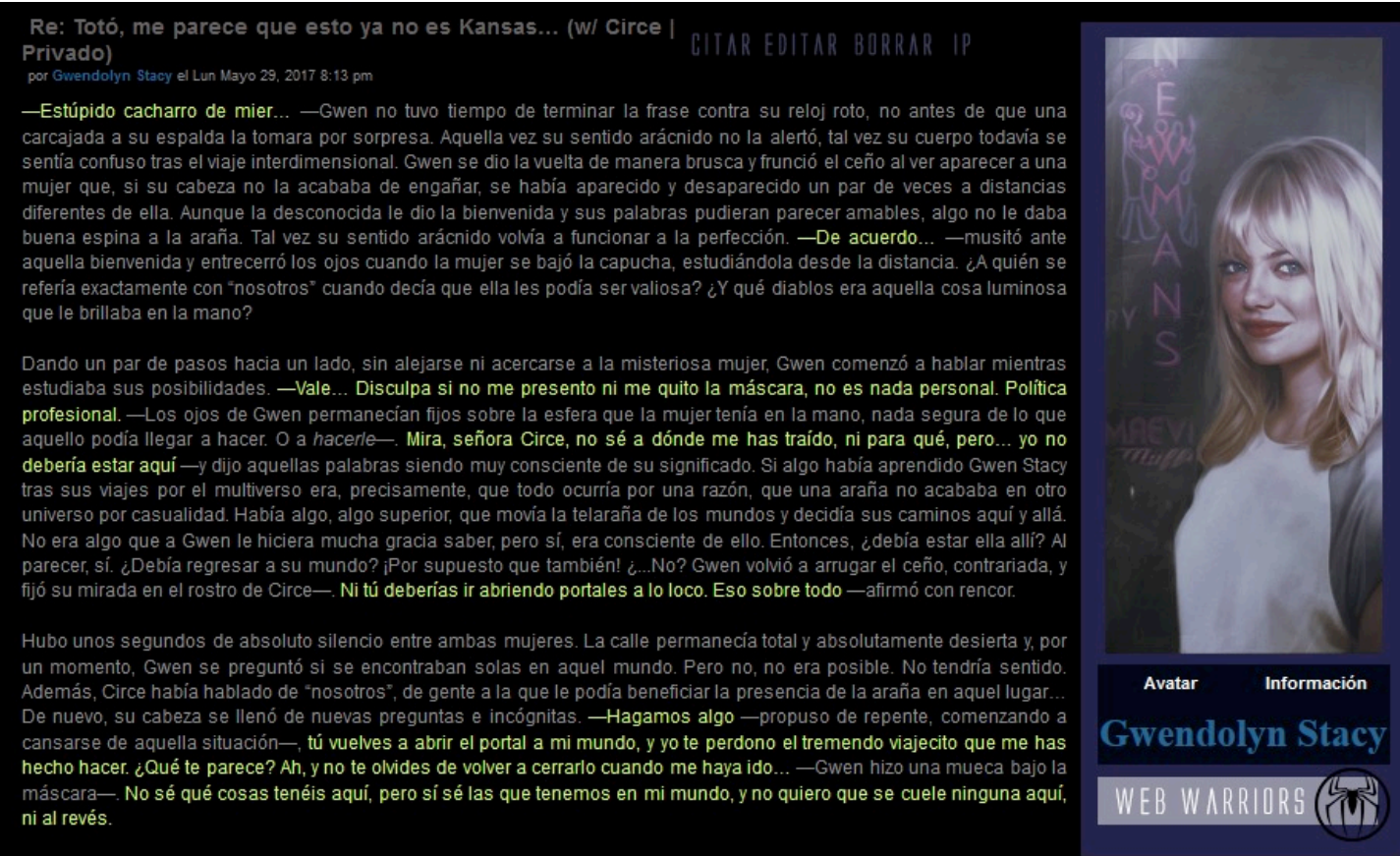

\section{time @if herdeg}

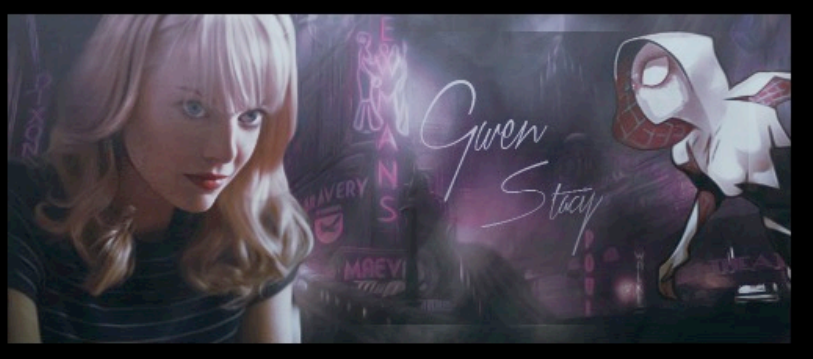

Ilustración 1: Ejemplo de mensaje de rol. 


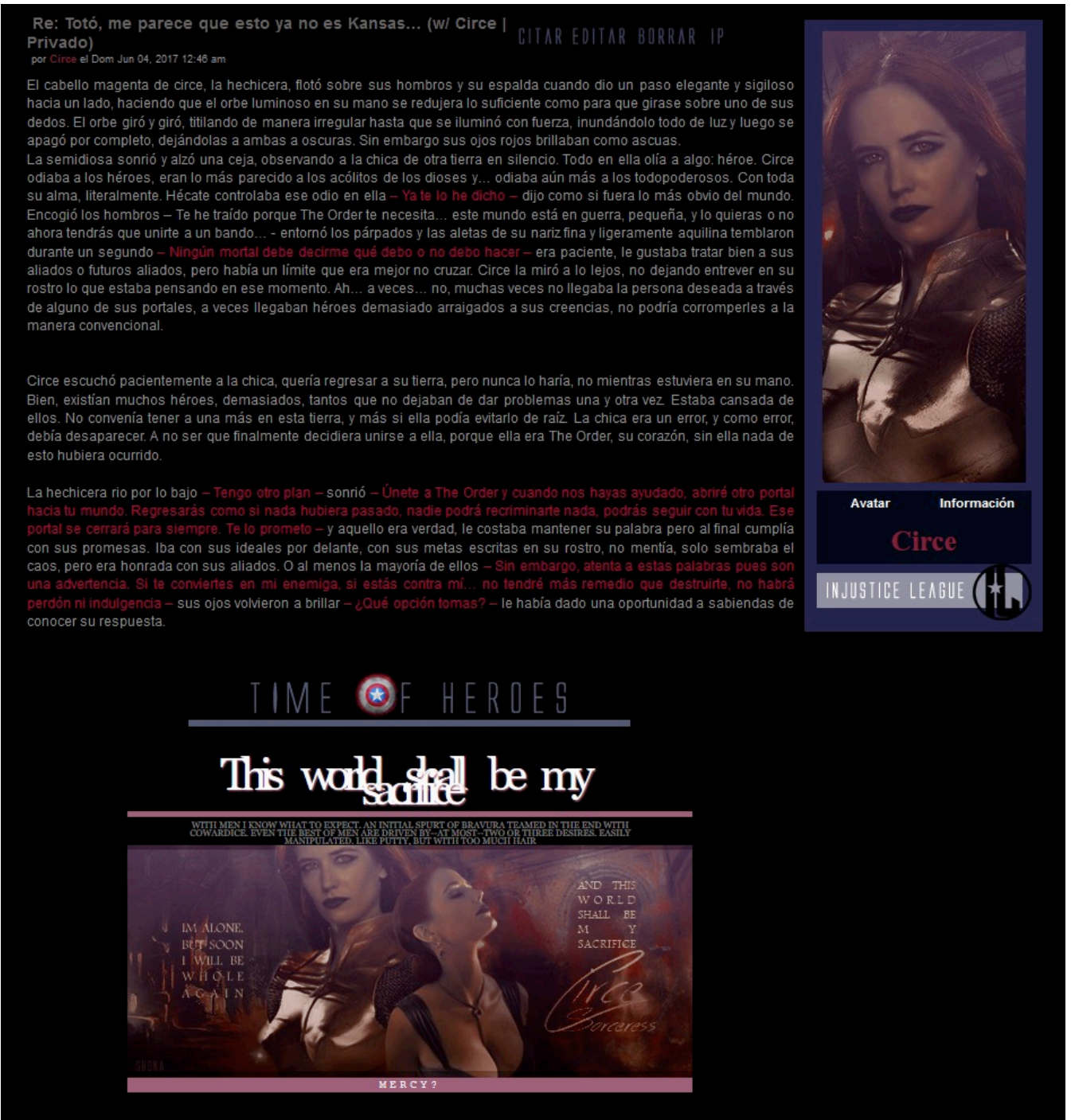

Ilustración 2: Ejemplo de mensaje de rol (reacción al mensaje de la Ilustración 1).

En estos ejemplos de mensajes de rol (Ilustración 1 y 2), observamos cómo se desarrolla esta interacción, que se produce a modo de respuesta al mensaje anterior. La narración, que contiene descripciones, detalles y sensaciones, combina también diálogos del personaje que el usuario está interpretando, de forma que se establece una conversación entre los personajes que participan dentro del hilo. Así pues, el play-by-post-rolepaying supone una perspectiva original para la escritura colaborativa y una nueva práctica innovadora para el tratamiento de la destreza escrita dentro de la enseñanza.

\section{La percepción de los usuarios de foros de rol sobre el desarrollo de su escritura}

Desafortunadamente, en la actualidad no disponemos de estudios e investigaciones acerca de los foros de rol aplicados a la enseñanza, por lo que el potencial de esta herramienta virtual para el desarrollo de la escritura y el aprendizaje de la destreza escrita no ha podido ser testado todavía. No obstante, con el fin de suplir esta carencia, hemos realizado un estudio de campo para aproximar los foros de rol al contexto de la enseñanza de segundas lenguas y descubrir de primera mano cuál es la percepción de aquellos usuarios que participan activamente en estas páginas acerca del desarrollo de su propia escritura a lo 
largo de su recorrido como participantes.

\subsection{Metodología de la investigación}

El trabajo de campo que presentaremos a continuación se compone de dos etapas muy marcadas y diferenciadas, puesto que para cada una de ellas se empleó un método de investigación distinto.

En primer lugar, se efectuó una fase preliminar en forma de online focus group con un número reducido de participantes, que compone la parte cualitativa de nuestra investigación. El objetivo principal de esta primera etapa era realizar una observación y recolección inicial de datos acerca de los foros de rol y de sus participantes, con el fin de diseñar la parte técnica de la siguiente fase. En este inicio de la investigación, se buscaba averiguar qué percepción tenía el grupo acerca del desarrollo de su propia escritura y la del resto en su lengua materna mediante el uso de los foros de rol. Al mismo tiempo, se trató de comprender el funcionamiento general de estas plataformas virtuales y las convenciones establecidas por los propios usuarios. A modo de cierre, se tanteó brevemente las impresiones de los participantes sobre la implementación de esta herramienta durante el aprendizaje de una segunda lengua.

Posteriormente, se inició una fase principal de investigación, basada en los resultados de la primera fase y con un enfoque más cuantitativo, en la que se elaboró y difundió un cuestionario con una cantidad mucho mayor de participantes y que, finalmente, nos permitió extraer una serie de conclusiones con el fin de profundizar en el potencial de la herramienta y su posible aplicación en la enseñanza de E/LE. La encuesta, procedimiento que se trabajó mediante Google Forms, trataba de conocer la percepción generalizada que los usuarios de foros de rol presentan acerca del posible desarrollo de su escritura a través de la plataforma.

De este modo, se persiguió obtener las impresiones de usuarios reales con el fin de conocer si estos percibían una mejora de su escritura a partir de su participación en foros de rol. A su vez, el cuestionario preguntó a los participantes su opinión acerca del posible uso de los foros de rol como herramienta de aprendizaje de una lengua extranjera. El estudio también trataba de ampliar la información recogida en la fase inicial (características de los foros de rol, mecánica y funcionamiento, experiencias individuales, etc.).

\subsection{Resultados de la fase preliminar: online focus group}

Para esta etapa inicial, se seleccionó a tres administradores de un foro de rol en habla hispana - lengua materna de los administradores - , puesto que se trataba de participantes con una amplia experiencia en el uso de esta herramienta. Este encuentro virtual se celebró a través de un chat en línea en el que los participantes fueron respondiendo a las preguntas de la moderadora a partir del siguiente guion:

\section{Presentación}

- Presentación y saludo de la moderadora para una primera toma de contacto.

- Exposición del tema a tratar y el motivo por el que se realiza el encuentro.

- Presentación de cada uno de los participantes.

\section{Descripción del procedimiento del focus group a los participantes}

- La conversación será incluida en un anexo del trabajo de investigación.

- Os pido que respondáis con sinceridad a cada una de las preguntas. En caso de que no 
estéis de acuerdo con la opinión de otro participante, es importante que nos lo comentéis.

- ¿Alguna pregunta?

3. Introducción al tema de debate (a partir de preguntas muy generales)

- ¿Os gusta rolear?

- ¿Cuándo roleáis?

- ¿Cuánto tiempo lleváis roleando en foros de rol?

- ¿En cuántos foros de rol habéis participado?

\section{Preguntas de transición}

- ¿Cuál es la parte que más disfrutáis de rolear?

- ¿Y la que menos?

- ¿Qué os suele motivar más: el personaje o la trama?

- ¿Cuántos personajes soléis llevar en un mismo foro?

- ¿Roleáis con personas desconocidas o preferís rolear con usuarios ya conocidos?

- ¿Creéis que habéis hecho amigos a través de los foros de rol?

5. Preguntas específicas sobre el tema de estudio (contraste entre su experiencia en el pasado y en el presente)

- ¿Cómo eran vuestros primeros personajes y tramas en los foros de rol?

- ¿Habéis releído algunos de vuestros posts antiguos?

- ¿Qué diferencias encontráis entre esos primeros personajes y tramas y las actuales?

- ¿Creéis que ahora tenéis más y mejores ideas que antes?

- ¿Soléis planear previamente las tramas que lleváis con vuestros personajes con los demás usuarios?

- Cuando escribís un post, ¿lo releéis antes de enviarlo?

- En cantidad, ¿escribís más líneas o menos que antes?

- ¿Y en calidad? ¿Os consideráis mejores rolers? ¿Por qué?

- En general, ¿pensáis que vuestra escritura ha mejorado con el paso del tiempo? ¿Por qué?

- ¿Habéis roleado alguna vez en un idioma que no era el vuestro?

- ¿Os gustaría intentarlo? ¿Por qué?

\section{Preguntas de cierre}

- ¿Recomendaríais a alguien que usara foros de rol? ¿Por qué?

- Un consejo para alguien que nunca ha roleado.

\section{Agradecimiento por la colaboración y despedida}

En esta reunión, los participantes compartieron sus experiencias y aportaciones acerca de diferentes aspectos relacionados con los foros de rol que permitieron extraer una serie de conclusiones sobre la actividad en la plataforma.

En primer lugar, los tres participantes compartían la impresión de que los foros de rol ocupaban un espacio importante en sus vidas; en los tres casos llevaban más de diez años dedicándole tiempo y esfuerzo a estas páginas, por lo que comprobamos que los foros de rol constituyen una herramienta que logra mantener el interés de sus usuarios durante más de una década.

Sobre el impacto que los foros de rol han causado en sus vidas se mencionaron distintos aspectos, como la capacidad de documentación en determinados casos para poder 
interpretar adecuadamente a un personaje, o el trabajo de la empatía al realizar el ejercicio de ponerse en la piel de una persona diferente a ellos mismos. En esta línea, los tres coincidieron en que la participación en foros de rol les había ayudado a «aprender a escribir», lo que nos permitió observar de manera inicial que eran conscientes de un posible desarrollo de la escritura.

Para ahondar en este aspecto, se dirigió la conversación con unas preguntas con el fin de que los participantes realizaran una comparación entre sus producciones en sus inicios dentro de foros de rol y sus escritos en la actualidad. En los tres casos apreciaban un gran cambio tanto en los textos como en los personajes y señalaban que ambos aspectos van un poco de la mano: trama y personaje evolucionan a un ritmo similar, pues la búsqueda de una mayor profundidad del personaje requiere una mejora del texto - mayor nivel de detalle y de emociones-.

En cuanto a los procesos de composición, se hizo mención tanto a la planificación como a la revisión de los textos. Sobre la planificación previa, afirmaron que varía en gran medida según el otro participante del hilo; antes de comenzar de lleno con el rol, los usuarios suelen mantener una conversación previa para discutir el desarrollo del hilo, y dependiendo de las necesidades o intereses de cada uno, se llega a un acuerdo. En general, los tres parecían concordar en que suele ser conveniente una planificación previa, aunque sea mínima y superficial. Por otro lado, sobre la revisión posterior del texto, los participantes presentaban comportamientos completamente distintos, por tanto nos encontramos ante procesos de escritura variados, lo que nos lleva a pensar que, efectivamente, cada escritor afronta sus producciones de manera única y personal, lo cual resulta muy interesante y enriquecedor para un aula de E/LE.

La conversación derivó en la percepción de una mayor calidad en los textos del foro a lo largo del tiempo, atendiendo a aspectos más lingüísticos y textuales: ortografía, gramática, orden del texto, puntuación, etc. Los tres administradores concluyeron sus aportaciones afirmando que se consideraban mejores escritores de lo que eran en el pasado gracias a todas las producciones que habían acumulado en foros de rol, y realizaron mención especial al resto de compañeros de rol, puesto que consideraban que el trabajo en equipo había sido una pieza fundamental para esta evolución.

Debido a su larga trayectoria en foros de rol, nos permitimos tantear sus impresiones acerca de la posibilidad del uso de esta herramienta dentro de un aula de E/LE. Ninguno de los tres se había aventurado a rolear en una lengua extranjera, aunque uno de ellos reconoció habérselo planteado en alguna ocasión a modo de aprendizaje extra. Todos coincidieron en que podía resultar de su interés en caso de que se encontraran estudiando una lengua diferente a su lengua materna.

\subsection{Resultados de la fase principal: trabajo de campo}

Tras recoger las primeras observaciones a través del online focus group, la investigación avanzó hacia la fase principal, cuyo principal objetivo se situaba en conocer la percepción generalizada que los usuarios de foros de rol presentaban acerca de la posible evolución de su escritura a través de la plataforma y, de manera paralela, analizar el funcionamiento de este tipo de foros y contrastar los diferentes intereses, opiniones y actitudes de sus usuarios.

Para alcanzar este objetivo, se partía de la hipótesis de que, efectivamente, los foros de rol funcionan como herramienta principal en el desarrollo de las destrezas escritas de sus 
usuarios. El estudio se llevó a cabo mediante un cuestionario, diseñado a partir de las conclusiones de la fase preliminar y que se difundió exclusivamente entre usuarios de foros de rol hispanohablantes. La muestra obtenida alcanza un total de 354 participantes; no obstante, se descartaron 5 sujetos tras detectar contradicciones en las respuestas proporcionadas, que denotaban falta de rigor en la participación, por lo que la cantidad de la muestra definitiva recoge 349 sujetos.

A pesar de que la selección de los sujetos fue aleatoria - es decir, no se eligió de forma personal e individual a cada uno-, pudimos trazar un perfil de los participantes a partir de unas preguntas iniciales de carácter sociológico. Así pues, la mayoría de sujetos fueron mujeres $(65 \%)$, seguidas de hombres $(32,1 \%)$ y una pequeña porción no se identificó con ninguno de estos dos grupos $(2,9 \%)$.

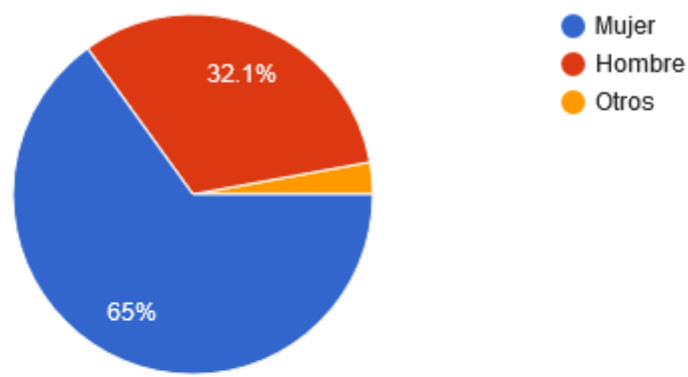

Ilustración 3: Baremo de género.

Respecto a la edad, resulta complicado estimar una aproximación general, puesto que obtuvimos resultados muy variados, lo que también nos permite comprobar que los foros parecen atraer a un rango de edad muy amplio: el 41,5\% de los sujetos tenían entre 20 y 25 años; el $31,5 \%$, entre 25 y 30 ; el $14,3 \%$, más de 30 ; el $11,7 \%$, entre 15 y 20 ; y, finalmente, un $0,9 \%$, menos de 15 .

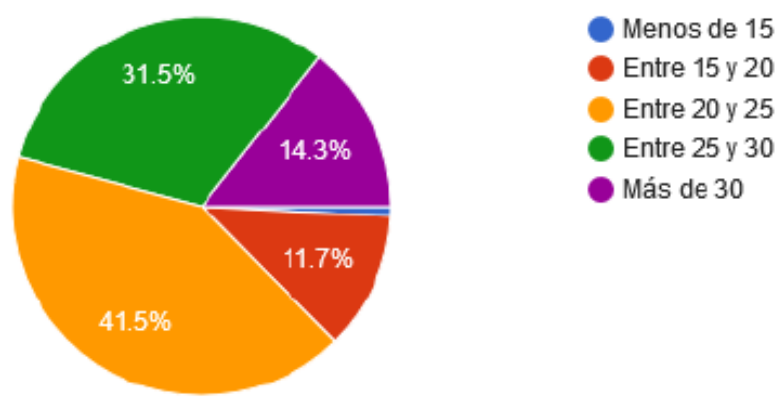

Ilustración 4: Baremo de edad.

En cuanto a la nacionalidad, un $63,3 \%$ de los participantes procedían de España, un $12,9 \%$ de México y un 9,7\% de Argentina, mientras que el 14,1\% restante se divide en porcentajes más pequeños, todos de países hispanos. Al preguntarles por su lengua materna, la gran mayoría respondió español (94,9\%). 

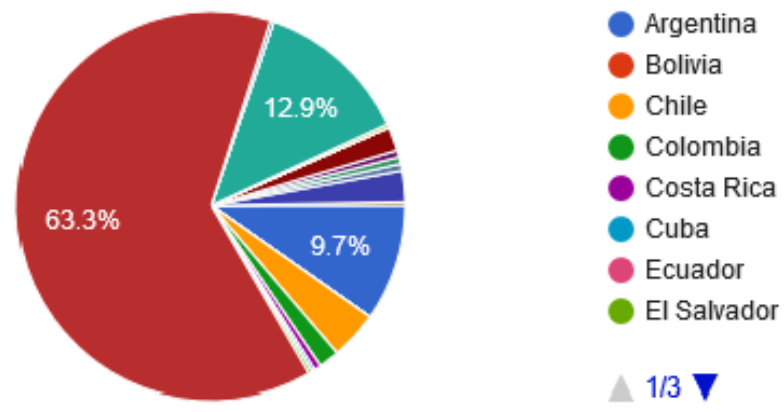

Ilustración 5: Baremo de nacionalidad.

Los datos obtenidos a través del cuestionario nos permitieron llegar a una serie de conclusiones que parten de la hipótesis inicial de la investigación. Así pues, en primer lugar, podemos corroborar que los usuarios de foros de rol perciben un desarrollo considerable en sus escritos a lo largo de su experiencia: consideran que sus producciones actuales presentan una mayor calidad que aquellas más antiguas - las ideas fluyen con mayor facilidad, la longitud de sus textos se ha extendido y distinguen una mayor profundidad y preocupación por los detalles - . Dentro de este desarrollo, destacan también la imaginación, creatividad e ideas, la profundidad del personaje, la narración de sucesos y el nivel de detalle de las descripciones realizadas. En general, se muestran orgullosos de sus producciones y reconocieron regresar a ellas cada cierto tiempo, por lo que distinguimos un importante papel del factor afectivo dentro de esta actividad.

Respecto a los procesos de escritura, como ya observamos en un primer momento durante la fase preliminar, comprobamos que, en efecto, varían notablemente de un participante a otro. No obstante, podemos extraer algunas conclusiones generales; por ejemplo, podemos afirmar que sí existe un cuidado por la presentación final de la producción escrita, puesto que apreciamos que la tendencia general de la mayoría de sujetos era realizar una revisión del texto antes de publicarlo. Por otra parte, en cuanto a la planificación previa de los hilos, obtuvimos respuestas muy diferentes; aunque sí se detecta una ligera inclinación hacia el lado de una puesta en común previa entre los usuarios implicados, también apreciamos una considerable cantidad de sujetos que prefieren dejar el desarrollo en manos de la improvisación, por lo que concluimos que es un aspecto muy personal de cada autor en el que juega un papel importante la interacción con el resto de participantes $\mathrm{y}$, por tanto, resulta complicado establecer una tendencia general.

Otra de las conclusiones más importantes del estudio reside en el claro fomento del trabajo colaborativo y de la interacción entre participantes que logran los foros de rol a través del planteamiento que se realiza de la producción conjunta de textos escritos. Los sujetos se muestran abiertos a rolear con desconocidos y afirman haber iniciado muchas amistades a partir de los foros de rol. También reconocen haber aprendido de otros compañeros y observamos que el hecho de que los escritos estén destinados a ser leídos y respondidos por otro usuario genera una especie de competencia sana que promueve que se muestren exigentes con sus propias producciones, por lo que detectamos un claro potencial de aprendizaje colaborativo dentro de la herramienta. 
Por otro lado, podemos afirmar que los foros de rol consiguen mantener la motivación sostenida de sus usuarios, a menudo durante largos periodos de tiempo: casi la mitad de los encuestados aseguraron llevar más de nueve años formando parte de comunidades de rol en línea. Asimismo, detectamos un número elevado de participantes que consideran que su participación en foros de rol les permite desconectar de otras actividades. Observamos, por tanto, que los foros se convierten en una herramienta de apoyo a nivel personal tal vez para individuos más introvertidos o sencillamente aquellos que desean aprovechar la escritura en foros como un momento de desconexión más personal.

De todos los resultados y conclusiones que pudimos extraer de esta investigación, la verdadera sorpresa sin lugar a dudas llegó con las preguntas acerca de la introducción de los foros de rol como herramienta de aprendizaje de segundas lenguas. Hacia el final del cuestionario, preguntamos a los participantes si alguna vez habían participado en un foro de rol cuya lengua vehicular fuese diferente a su lengua materna. Los resultados desvelaron que una importante cantidad de usuarios habían probado en algún momento el rol en una lengua extranjera - la mayoría, inglés - . Un gran número afirmó que esta práctica había contribuido a mejorar su nivel en la lengua extranjera; en algunos testimonios, se señaló que el cambio exigió un trabajo extra, puesto que la expresión en una lengua distinta a la materna puede ser compleja, pero parecen coincidir en que los resultados merecieron el esfuerzo.

Por otra parte, los usuarios que nunca habían roleado en una lengua extranjera se dividieron entre los que se mostraban más abiertos hacia la posibilidad de intentarlo en algún momento, aunque observamos una preocupación por no considerar que poseen el nivel suficiente en la segunda lengua, por lo que se sienten intimidados ante la idea; y, por otro lado, los usuarios que se cerraban por completo a ello, bien por falta de nivel, confianza, tiempo o incluso recursos.

\section{De la teoría a la práctica: los foros de rol en el aula de $\mathrm{E} / \mathrm{LE}$}

Los resultados de la investigación originaron nuevas cuestiones: ¿cómo puede encajar el foro de rol como parte del aprendizaje dentro de un curso de E/LE? ¿Qué puntos fuertes presenta una propuesta de estas características? ¿En qué aspectos puede flaquear? Desgraciadamente, como nuestro estudio no ha contado con la posibilidad de llevar a la práctica una propuesta didáctica cuyo eje central fueran los foros de rol, no podemos basar nuestra reflexión en resultados reales de una puesta en práctica.

No obstante, para poder analizar las diferentes cuestiones que surgen a la hora de implementar el foro de rol dentro de la enseñanza de E/LE, hemos realizado una reflexión acerca de su uso basándonos en los resultados obtenidos durante nuestro estudio de campo y apoyándonos en cuestiones más teóricas. En esta reflexión, aportamos una serie de beneficios que este tipo de plataformas pueden proporcionar para el aprendizaje de E/LE; al mismo tiempo, presentamos posibles inconvenientes que pueden presentarse a lo largo del desarrollo activo de una propuesta didáctica. En relación a estos últimos, hemos acompañado posibles opciones para solventarlos.

\subsection{Ventajas}

En primer lugar, al realizar una comparación entre los foros de rol y el resto de propuestas $y$ actividades que se han empleado hasta ahora dentro de la enseñanza de E/LE, observamos que esta herramienta presenta una práctica de escritura colaborativa muy diferente e innovadora. Este tipo de rol nos ofrece una alternativa a las producciones 
escritas más tradicionales, abordando la escritura desde una perspectiva más lúdica.

El foro de rol dentro de una propuesta didáctica no solo ofrece una revolución para las actividades de producción escrita, sino también para el tipo de herramientas con las que trabajan los aprendientes de E/LE. En muchas ocasiones, dentro de los cursos de E/LE, los docentes no consiguen sacar todo el partido posible de las herramientas digitales, ya sea por falta de tiempo, conocimiento o recursos. Esta propuesta se beneficia especialmente de las TIC e Internet, presentando una explotación de los recursos digitales de los que disponemos en la actualidad.

Por otra parte, al trabajar las producciones a través de un foro, el aprendiente puede ser más consciente de su evolución y de sus errores lingüísticos de lo que lo sería en otro tipo de actividades de rol, más enfocadas en el habla oral. Los textos quedan registrados en la plataforma, completamente accesibles tanto para los aprendientes como para el docente, lo que facilita llevar un control sobre aquellos aspectos en los que el aprendiente considera que debe mejorar. De esta forma, podemos llegar a utilizar los hilos de rol como un corpus con el que trabajar la lengua desde una perspectiva, por ejemplo, más gramatical. No obstante, nuestro estudio considera que el funcionamiento y las mecánicas que se establecen en los foros de rol favorecen un enfoque didáctico de la producción escrita más basado en los procesos de composición que otras metodologías, como pueden ser el enfoque basado en la gramática o el enfoque basado en funciones (Cassany, 1990). La propuesta puede servir a los intereses de cualquier tipo de escritor, independientemente de cómo sea su proceso de composición, o incluso ayudar al aprendiente a crear un hábito de escritura si todavía no lo tiene.

El fomento del aprendizaje colaborativo y de la interacción es uno de los aspectos clave de esta herramienta, y como hemos observado durante nuestro estudio, un factor importante para los usuarios activos de los foros de rol. Este fenómeno nos recuerda a la teoría sociocultural de Vygotsky (1978), que afirmaba que el desarrollo cognitivo es menos biológico y más social, y pone de manifiesto la importancia de la interacción y del componente social dentro del trabajo en el aula para el desarrollo del aprendizaje. Como ya apreciamos en los resultados de la investigación, los participantes de los foros afirman haber aprendido gracias a sus compañeros de rol y de realizar un mayor esfuerzo en su trabajo para, de alguna forma, «estar a la altura» de sus compañeros. Paralelamente, el foro puede servir al docente como método para mejorar el ambiente dentro de su grupo y promover así la interacción entre aprendientes y reforzar las relaciones en el aula, se conozcan previamente o no.

El planteamiento del foro de rol dentro de una propuesta de E/LE promueve también el aprendizaje autónomo, puesto que los aprendientes deben encargarse de administrar su propio tiempo y trabajo en función de sus necesidades o circunstancias. Asimismo, convierte al alumno en protagonista de la actividad, mientras que el profesor permanece en un segundo plano, ocupando el papel de guía y moderador.

Dado que el trabajo se realiza a través de una herramienta asincrónica de comunicación, el aprendiente puede gestionar su tiempo y actividad como mejor le convenga, lo que convierte a los foros de rol en una plataforma aplicable tanto en cursos presenciales como a distancia. Las características y el funcionamiento de la página benefician especialmente a este segundo tipo de cursos, donde los estudiantes pueden encontrarse en localizaciones geográficas muy distintas, una situación que a menudo dificulta la realización de actividades conjuntas. 
Por último, no podemos olvidar el gran potencial que manifiesta la plataforma de rol en relación a la motivación, un aspecto tan importante a la vez que complejo dentro de la enseñanza. A menudo resulta complicado plantear actividades de producción escrita sin sufrir el desinterés de los aprendientes; el foro de rol se presenta como una herramienta diferente, original y más moderna que puede despertar el interés del grupo y conseguir mantenerlos motivados para enfrentarse a la producción escrita desde una perspectiva más creativa e innovadora. El uso de la plataforma puede cambiar la visión de ciertos aprendientes sobre la escritura en español, o incluso del aprendizaje de E/LE en general.

\subsection{Inconvenientes}

Para implementar con éxito los foros de rol dentro de la enseñanza de E/LE, debemos también atender a los inconvenientes que puede conllevar el uso de la herramienta o las dificultades que pueden surgir a lo largo de su empleo y tratar de solventarlos de antemano.

Por una parte, el principal obstáculo con el que el docente puede encontrarse radica en la falta de medios necesarios para implementar la propuesta, es decir, el acceso a ordenadores e Internet. En el caso de cursos presenciales, es posible que el aula donde se imparta el curso no cuente con estos recursos. Tal vez por este motivo los foros resultan una herramienta tan interesante para cursos a distancia o en línea. Aun así, no se descarta en absoluto la posibilidad de implementarlos en un curso presencial; el docente podría convertir la falta de herramientas informáticas en una buena oportunidad para trasladar la práctica de rol a tareas extra que se realicen fuera del aula, de modo que el foro sirviera de refuerzo para practicar todos aquellos contenidos que ya se trabajan dentro del aula.

Como ya señalábamos anteriormente, el docente ocupa dentro de esta propuesta un lugar en segundo plano; no obstante, la actividad requiere que el profesor observe una cantidad numerosa de trabajos y grandes extensiones de texto y, por tanto, exige del docente mucho tiempo del que quizá no disponga. Para solucionar este problema, se puede plantear la revisión entre compañeros; es decir, puesto que los aprendientes deben leer los escritos del compañero con el que rolean para poder reaccionar y producir sus textos a modo de respuesta, la evaluación por parte del compañero resulta hasta natural. De este modo, la autoevaluación y la evaluación del compañero se convierten en factores fundamentales de la composición vista desde el enfoque basado en el proceso, cuyas fases recogen la planificación, la textualización $\mathrm{y}$, finalmente, la revisión de la producción escrita (Cassany, 2005: 42-43).

Otro posible inconveniente para la incorporación de la herramienta lo encontramos en la competencia digital, cuando se presenta una profunda carencia de conocimientos. Puede darse por parte de los aprendientes, en casos en los que estos procedan de un contexto en el que la tecnología no se encuentre tan presente como en otros países. También puede manifestarse por parte del docente, quien tal vez no cuente con las habilidades informáticas necesarias para desenvolverse adecuadamente con la plataforma. No obstante, es necesario señalar que en la actualidad existe una gran variedad de portales digitales que ofrecen la posibilidad de crear foros a través de una interfaz muy sencilla e intuitiva, además de numerosos tutoriales en la red que pueden ayudar al trabajo tanto de los aprendientes como del propio docente, por lo que con un poco de tiempo y trabajo se puede llegar a suplir esta carencia con diferentes apoyos.

Para finalizar, hemos de tener presente que, por más que los foros de rol se introduzcan en la clase de E/LE con una propuesta bien organizada y con entusiasmo, cabe la 
posibilidad de que la propuesta no termine de cuajar con un grupo determinado, ya sea por falta de interés u otros motivos más personales. Por esta razón, resulta muy importante que el profesor sepa escuchar a sus alumnos, se moleste en conocer sus intereses, expectativas y necesidades para lograr adaptar la propuesta y motivar verdaderamente al grupo en cuestión.

\section{Conclusiones}

Con el transcurso del tiempo se vuelve cada vez más evidente que la era digital y la enseñanza deben avanzar de la mano y que, sin lugar a dudas, tienen mucho que aportarse mutuamente. A través de nuestro estudio, hemos tratado de abrir nuevas líneas de investigación que respondan a esta realidad digital y que a su vez puedan aplicar las metodologías correspondientes. La presente investigación buscaba dar respuesta a multitud de interrogantes; si bien todavía quedan muchos por descubrir, hemos logrado llegar a una serie de conclusiones.

En primer lugar, podemos establecer que los foros de rol han demostrado poseer un gran potencial dentro del campo de la enseñanza de idiomas. Esta herramienta destaca por ofrecer diferentes características que resultan altamente beneficiosas para el aula de E/LE: gran versatilidad, aprendizaje colaborativo a la vez que autónomo, motivación sostenida, control de los errores, interacción en línea, etc. El estudio de campo ha permitido corroborar que la gran mayoría de usuarios de foros perciben un considerable progreso de su escritura gracias a su experiencia en el mundo del rol, lo cual abre la puerta a la posibilidad de que esta herramienta se convierta en un recurso atractivo y eficaz para los aprendientes de E/LE. Al mismo tiempo, dicha investigación nos ha desvelado que un número considerable de usuarios rolean o han roleado alguna vez en un idioma diferente a su lengua materna, por lo que observamos que esta práctica ya se realiza dentro de un hábito de aprendizaje de idiomas más autónomo.

Ante la posibilidad de elaborar una propuesta didáctica basada en el uso de los foros de rol como herramienta para desarrollar las destrezas escritas en E/LE, la investigación se decanta por un enfoque didáctico basado en el proceso de producción escrita. De este modo, consideramos que el aprendiente logrará trazar su propio hábito de escritura, convirtiéndose en un buen escritor crítico y, además, conociéndose mejor a sí mismo tanto como escritor como individuo-.

Asimismo, el presente estudio intenta establecer una base a partir de la que comenzar otro tipo de investigaciones que puedan ahondar en la herramienta presentada dentro de la enseñanza de E/LE desde una perspectiva más práctica, así como también trasladarla a otros campos de investigación. El potencial de nuestro objeto de estudio puede trasladarse a otros ámbitos dentro de la propia educación — como materias más relacionadas con la literatura o la historia-, o también fuera de ella — campos relacionados con las TIC, como pueden ser la programación o el diseño gráfico- - En la actualidad, los foros de rol constituyen toda una herramienta por descubrir que sin duda animamos a continuar estudiando, descifrando e incorporando en las prácticas docentes.

\section{Bibliografía}

CAlzadilla, M. E. (2002). «Aprendizaje colaborativo y tecnologías de la información y la comunicación». Revista Iberoamericana De Educación. Vol. 29, n. ${ }^{\circ} 1$. Recuperado el 14/8/19, de: https://rieoei.org/RIE/article/view/2868 
CASSANY, D. (1989). Describir el escribir. Barcelona: Paidós.

CASSANY, D. (1990). «Enfoques didácticos para la enseñanza de la expresión escrita». Comunicación, lenguaje y educación. Vol.6, pp.63-50. Recuperado el 14/8/19, de: https://dialnet.unirioja.es/descarga/articulo/126193.pdf

CASSANY, D. (2005). Expresión escrita en L2/ ELE. Madrid: Arco / Libros.

CONSEJO DE EUROPA (2018). Common European Framework of Reference for Languages: Learning, Teaching, Assessment. Companion Volume with New Descriptors. Recuperado el 14/8/19, de: https://rm.coe.int/cefrcompanion-volume-with-newdescriptors-2018/1680787989

Csenge, Z. (2017). Collaborative Storytelling 2.0: A Framework for Studying Forum-

Based Role-Playing Games (Tesis de doctorado). Bowling Green State University: Harvard University Press. Recuperado el 14/8/19, de: https://pdfs.semanticscholar.org/7d4c/15b221e468a1b0dd2e4b24b59c30583df0dc.pdf

MolÉs, M. (2018). El desarrollo de la destreza escrita en ELE: los foros de rol como herramienta de aprendizaje (Trabajo de Fin de Máster). Recuperado el 14/8/19, de: https://repositorio.unican.es/xmlui/bitstream/handle/10902/15540/TFM.MMM.pdf

RAIMES, A. (1991). "Out of the Woods: Emerging Traditions in the Teaching of Writing». TESOL Quarterly. Vol. 35, núm. 3, pp. 407-430. Recuperado el 14/8/19, de: http://www.personal.psu.edu/kej1/APLNG_493/old_site/raimes.pdf

Vygotsky, L.S. (1978). Mind in Society: The Development of Higher Psychological Processes. Cambridge: Harvard University Press. 